\title{
Effect of Climate Change on Mental Health
}

\author{
Dr. Ratan P. Solanki ${ }^{1 *}$
}

\section{ABSTRACT}

On $5^{\text {th }}$ of June the entire world celebrated the World Environment Day (WED) WED is being celebrated every year since 1973.To involve people all over the world, a slogan has also been coined for this year's WED. The slogan is: 'Raise Your Voice Not the Sea Level.' The idea is that there is need to raise voice against the ongoing climate change so that brake is applied to the raising global temperature. 23We all know that 2014 has been declared as the hottest year globally by the Meteorological department of United States of America. Climate change is a global challenge which is likely to affect the mankind in substantial ways. Not only climate change is expected to affect physical health, it is also likely to affect mental health. Increasing ambient temperatures is likely to increase rates of aggression and violent suicides, while prolonged droughts due to climate change can lead to more number of farmer suicides. Droughts otherwise can lead to impaired mental health and stress. Increased frequency of disasters with climate change can lead to posttraumatic stress disorder, adjustment disorder, and depression. Changes in climate and global warming may require population to migrate, which can lead to acculturation stress. It can also lead to increased rates of physical illnesses, which secondarily would be associated with psychological distress. The possible effects of mitigation measures on mental health are also discussed. The paper concludes with a discussion of what can and should be done to tackle the expected mental health issues consequent to climate change.

Keywords: Climate Change, Mental Health, Stress, WED

Human influence has been the dominant reason behind the observed warming of climate since the mid- $20^{\text {th }}$ century. Increased exposure to heat is likely to become more common with the rise in the global temperatures. It has been suggested that there is a relation between temperature rise and aggressive behavior. Increase in rates of criminality and aggression have been observed during the hot summer months, suggesting a link between aggressive behaviors and temperatures. With global warming, it is possible that the rates of aggression may increase over time. Association has been also been seen with the rates of suicides and the temperatures. It has been seen that suicides, especially violent ones are more common with the recent increase in

\footnotetext{
${ }^{1}$ Shree Mahila Arts \& Home Science College, Kodinar, Gujarat, India *Responding Author

(C) 2016 I R Solanki; licensee IJIP. This is an Open Access Research distributed under the terms of the Creative Commons Attribution License (http://creativecommons.org/licenses/by/2.0), which permits unrestricted use, distribution, and reproduction in any Medium, provided the original work is properly cited.
} 


\section{Effect of Climate Change on Mental Health}

temperatures Heat waves have been associated with mental and behavioral disorders. A study from Australia suggests that heat waves are associated with increased rates of admissions for mental disorders also, in conjunction with other disorders such as cardiovascular and renal illness. Such heat waves have been associated with mood disorders, anxiety disorders, dementia and anxiety related disorders among others. Extreme heat exposure can lead to physical as well as psychological exhaustion. A study from Thailand suggests that occupational heat stress is associated with greater psychological distress among the workers. Similar other studies have found an association between increased temperatures in the work place and greater psychological distress.

\section{Psychological consequence due to climate related disasters}

Climate related disasters such as floods, hurricanes, and bush-fires are often associated with stress-related psychiatric disorders. Individuals who have been exposed to life threatening situations are at a considerable risk of developing posttraumatic stress disorder (PTSD).The symptoms of PTSD include flashbacks of the event, increased arousal and avoidance of cues to the memory of the event. In many cases, the symptoms of PTSD may have a delayed onset, months to years after the experiencing of threatening disaster situation. Development of PTSD is associated with impairment in the quality of life and significant subjective distress.

Individuals who have been through the experience of climate related natural disaster are not only at a higher risk of developing PTSD, but also at a greater risk of developing acute stress reaction and adjustment disorder. These disorders are anxiety spectrum disorders which can subside over a period of time with rehabilitations and/or treatment. Other stress exacerbated disorder includes development of acute and transient psychosis and relapse of bipolar disorder. Faced with the loss of home, environment, social structures and loved ones, an individual may develop a bereavement (grief reaction) or depression. The depression is likely to be more pronounced in those who live in small rural communities, than those living in big cities. As the impact of climate change seems to be increasing over the time period, it is likely that a greater proportion of the population would be impacted by the mental health consequences of climate change related disasters.

\section{Drought and farmer suicide}

Global climate change is likely to exacerbate droughts in the years to come. Changes in precipitation patterns are likely to lead to increased floods in some areas while prolonged droughts are expected in other areas. A relationship has been found between the occurrence of drought and farmer suicides. Such a trend has not only been found in developed country like Australia, but also in developing the country like India. Association has been found between crop failures due to unexpected droughts and suicide attempts in the farmers. Failure of crop can lead to economic hardships. When dependent on low precipitation situations, the farmer might not be able to sustain the expenses of the family and may become a victim of the debt trap to meet the 


\section{Effect of Climate Change on Mental Health}

expenses. Second, it may also lead to rise in expenses of food and other goods in the region. Inability to make basic purchases can lead to malnutrition and risk of other infections, especially in developing countries where structural social services are not efficiently organized. Third, droughts are also often associated with prolonged exposure to warm, dry season. As above, it seems likely that exposure to heat can lead to increased rates of suicide attempts. Fourth, prolonged droughts can lead an individual to migrate to another region and/or pursue another vocation. This leads to acculturation stress which may further lead to suicide attempts in the farmer population. Since much of the world population depends on the farmers for their food supply, health care of the farmers is an important issue and efforts are required to provide help to them when needed. Moreover, since the majority of the farmers live in rural areas while healthcare facilities are concentrated in urban areas, efforts are required for easy access to services to this population.

\section{Economic changes due to climate change and effect on mental health}

Societies dependent on agriculture are likely to be quite impacted by the changing climate. Agricultural land may be encroached upon by rising sea levels, desiccation or flooding. Moreover, extreme heat makes agricultural work less productive due to fatigue of the workers. Decreasing agriculture produce also hampers the production in agricultural support industries which also employ the manual laborers during the lean season. These can lead to economic hardship which can result in an increase in mental health problems. It has been observed that drought prone areas are vulnerable to lower socioeconomic status and higher levels of distress and helplessness. Long duration droughts have been associated with deterioration of economic conditions, which has been associated with depression and demoralization. Distress due to prolonged droughts have been found in adolescents and have been seen to increase with time.

Social capital which combines social cohesion and community participation is strained under economic pressure situations. Decrement in social capital can lead to a reduction in wellbeing and may influence genesis of mental health problems. Women are more likely to be affected than men with the reduction of social capital especially when they have to migrate for employment or other reasons, which is likely to secondarily impact the family wellbeing.

Economic constraints can also have an adverse impact of healthcare seeking, especially for mental health. The ability of the society to provide treatment may be reduced during periods of economic hardships. Individual's payment for treatment, which is the more common mode of payment of treatment in developing countries, can be affected due to economic adverse situations, leading to inadequate treatment opportunities and suboptimal treatment.

\section{Migration and acculturation stress}

Climate change is likely to be related to changes in habitat and ecosystems all over the world. Submergence of coastal areas, hurricanes and floods, and prolonged droughts are likely to be 


\section{Effect of Climate Change on Mental Health}

associated with migration of population, regionally and internationally. Previous mental health literature suggests that migration of individuals is related to acculturation stress, which is likely to act in the genesis of psychiatric disorders. For example, migrants are more likely to suffer from schizophrenia than the host population or the population of their origin. It has been suggested that the reasons of migration also influence the propensity to develop psychological problems in individuals. Those individuals forced to migrate after strife and disasters are more likely to suffer from psychiatric illness as compared to those individuals who choose to migrate voluntarily.

Usually, individuals develop a feeling of connectedness to their environment of residence. "Solastalgia" describes a loss of solace that occurs with the degradation of the environment of an individual's belonging. This discomfiture is likely to occur with the climate change resulting in changes in ecological balances and changing physical and climatic conditions in large parts of the globe.

\section{Association with physical illnesses}

Mental health is intricately linked with physical health. Poor physical health and ailments can lead to poor quality of life and psychological distress Often the psychological distress elicited by medical illnesses do not qualify for a severe psychiatric illness but require the diagnosis of adjustment disorders. Nonetheless, the anxiety and depressive symptoms generated as a consequence of physical illness require attention and are helped with treatment with antidepressants and counseling.

It is rarely in doubt that many physical illnesses would see increasing trends with climate change. Heat, drought, and flood related events are likely to be associated with increased rates of cardiovascular disorders, respiratory, gastrointestinal disorders, and renal problems. Environmental determinants such as pollen, smoke, dust, and a stagnant water consequent to heat, drought-related fires, and floods are likely to adversely affect human health and lead to chronic physical diseases. Occurrence of chronic physical disorders is likely affect mental health directly or indirectly due to strain on coping.

Climate change is also expected to lead to decrement in the overall arable land. This is likely to lead to a shortage in food supply if methods of boosting food productivity are not found. Malnutrition especially among children is likely to be exacerbated in developing countries if adequate food supply cannot be ensured with climate change. Nutritional deficiencies are likely to be associated with mental health problems like depression and cognitive decline.

\section{Effect of adaptation and mitigation measures}

Adaptation and mitigation measures aim to make individual adept to the changing environment and attempt to reduce environmental change in the future, respectively. Such measures by 


\section{Effect of Climate Change on Mental Health}

themselves may lead to change-related mental health risks through various causal pathways. For example, adaptation to the work situation can have some risks to mental health. For places which do not have air-conditioners, increase in ambient temperatures might lead to decreased productivity in the day time when the temperatures are high. For workers who are paid subsistence rates in developing countries, avoidance of work in excess heat may result in reduced incomes and growing poverty. If they attempt to compensate with extending their work hours or at night, it may impair their family and social relations, leading to reduced buffer for development of mental illness.

Similarly, traveling between continental cities through trains and buses for the purpose of work may reduce the carbon emissions due to airlines. However, it may result in time expense and less actual time for the business purpose. This might also mean greater time spent on travel which could have been rather utilized with other family members or friends, or for cultivating recreation. Having lesser time for social interaction in a familiar and desirable situation is likely to have an impact on mental health secondarily.

\section{What needs to be done?}

Since climate change is likely to impact human mental health in many ways, it is imperative that some steps are taken to either reduce the global warming with time or develop measures to deal with the challenges posed through adaptation. Mitigation of greenhouse gases involves less reliance on fossil fuels, developing and using alternate efficient power sources, reducing encroachment on green cover and other similar measures. There is a developing global perspective about the need to reduce the carbon footprint per person over the next few decades, and to cover the inequities between the rich and the poor countries. Countering the challenge of climate change requires inter-sect oral and international collaboration to implement policies for reducing the emission of greenhouse gases.

Developing countries like India have also developed and articulated their policies toward challenging the impact of climate change. The National Action Plan on Climate Change (NAPCC) documents the Indian government's plan to deal with the issue of climate change. The eight missions focused on by NAPCC involves National Solar Mission, National Mission for Enhanced Energy Efficiency, National Mission on Sustainable Habitat, National Water Mission, National Mission for Sustaining the Himalayan Ecosystem, Green India Mission, National Mission for Sustainable Agriculture, and National Mission on Strategic Knowledge for Climate Change. Each of the missions aims at mitigating the process or reducing the impact of climate change. The effect of implementation of these policies needs to be seen.

Meanwhile, provision of adequate treatment facilities for managing mental health problems should be undertaken. This is especially required for natural disaster-related problems, when the vulnerability to stress is acute. It is likely that the existing infrastructure of treatment might be 


\section{Effect of Climate Change on Mental Health}

compromised during the disaster situation. Such a time requires the inputs and help from professionals from other regions.

Promoting positive mental health is another way to mitigate the psychological distress due to climate change. Human resilience and coping can reduce the effect of mental health stress due to climate change. Utilization of strategies like yoga can be indigenous and acceptable ways to deal with stress.

Another method to reduce suicide fatalities due to secondary consequences of climate change may include debt-abolition or economic support for farmers. Creating co-operatives and protection of farmers from loan sharks might reduce the suicide rates due to crop-failures. Furthermore, provision of subsidies and guaranteed income during the drought seasons might lead to less economic and psychological stress on farmers in question.

What can and needs to be done in response to climate change can have many viewpoints. It might be probably useful to amalgamate the best from different solutions to provide a coherent, implementable and effective response to the concerns raised by climate change. And the solutions would be best refined with the systematic evidence accumulated over the course of time

\section{CONCLUSIONS}

Climate change is likely to affect mental health in many ways. Droughts, floods, rising sea level, increasing ambient temperatures and other consequences of climate change can produce increasing psychological distress through many mediators. These mediators include economic strain, migration and acculturation stress, lowering social capital, and traumatic events among others. Efforts to increase access to mental health services and attempts to mitigate the climate change with time would be appropriate responses to deal with the challenge of climate change in the time to come.

\section{Acknowledgments}

The author appreciates all those who participated in the study and helped to facilitate the research process.

\section{Conflict of Interests}

The author declared no conflict of interests.

\section{REFERENCES}

Pilkey OH, Cooper JA. Climate. Society and sea level rise. Science. 2004;303:1781-2.

Berry HL, Bowen K, Kjellstrom T. Climate change and mental health: A causal pathways framework. Int J Public Health. 2010; 55: 123-32 


\section{Effect of Climate Change on Mental Health}

Le Houérou HN. Climate change, drought and desertification. J Arid Environ. 1996; 34: 133-85. Deshpande RS. Suicide by farmers in Karnataka: Agrarian distress and possible alleviatory steps. Econ Polit Wkly. 2002;37:2601-10.

Sarma EA. Is rural economy breaking down? Farmers' suicides in Andhra Pradesh. Econ Polit Wkly.2004; 39: 3087-9.

Bhugra D, Jones P. Migration and mental illness. Adv Psychiatr Treat. 2001; 7: 216-22.

Rosswall T. Greenhouse gases and global change: International collaboration. Environ Sci Technol.1991; 25: 567-73.

Pandve HT. India's national action plan on climate change. Indian J Occup Environ Med. 2009; 13: $17-9$

Anderson CA. Heat and violence. Curr Dir Psychol Sci. 2001; 10: 33-8.

How to cite this article: R Solanki (2016), Effect of Climate Change on Mental Health, International Journal of Indian Psychology, Volume 3, Issue 4, No. 58, ISSN 2348-5396 (e), ISSN: 2349-3429 (p), DIP: 18.01.054/20160304, ISBN: 978-1-365-24976-1 